\title{
The Memory Promoting Effect \\ of Smiling Face in Face Cognition
}

\author{
Ningxia Wang \\ School of Law, Southwest University of Science and Technology \\ Mianyang 621010, Sichuan, China \\ Tel: 86-816-608-8322 E-mail: ningxia513@126.com
}

\begin{abstract}
This paper takes into consideration the universality of smiling face cognition and adapts holistic recognition strategy to study smiling face recognition within intercrossing culture. Research results showed that when the faces are upright, compared with quiet face, the response time of smiling face is quicker and the recognition time of smiling is improved. Moreover, holistic recognition strategy had its advantage under the upright condition; however it was uneasy to exert its function under the upside-down condition. Smiling face cognition was greatly influenced by the upside-down effect and smiling face recognition relied more on the holistic recognition strategy. What is more, holistic recognition strategy could overcome difficulties of recognizing various particular facial expressions of people from different cultures, and the smiling face could be commonly shared between the different cultures.
\end{abstract}

Keywords: Facial recognition, Upside-down effect, Smiling face, Holistic recognition strategy

\section{Introduction}

For the recognition of facial expressions, researchers have raised many recognition methods. Currently, the recognition methods of human beings' facial expressions could be divided into two types, namely, the one based on partial features and the one based on holistic features. The facial expression recognition based on partial features makes feature extraction according to the differences of the locations, sizes and mutual location differences of individual facial features (eyebrows, eyes, nose, mouth and face outline, etc.) to achieve facial expression recognition. The recognition based on the holistic features of faces applies the holistic face picture to extract the features that reflect face's holistic features so as to realize facial expression recognition (Maja, 2000)

The upside-down effect of Yin (1969) shows that when the examination series and recognition items are all upright pictures, the least the errors on facial stimulation recognition occur. However, when the examination series are upright pictures and the recognition items are upside-down pictures, the most the errors on facial stimulation recognition occur. Yin analyzed the oral reports of the examinees and found that the examinees applied holistic impression strategy to recognize upright faces while applied partial feature strategy to recognize upside-down faces. Combined with the right hemisphere of brain advantages phenomenon (Yin, 1970), Yin alleged that facial recognition system has specialty.

Carey and Diamond (1977) pointed out that the differences between upright and upside-down recognitions are qualitative differences. Under the condition of upside-down, the configurable information of face is destroyed, which results in poor recognition effect.

Valentine (1988) pointed out in the review of the upside-down effect of face that besides the factor that the examinees turned from holistic strategy to partial strategy in the process of face recognition, the factors that cause faces to be hard to recognize may also include facial expression, prototypes of faces, the familiarity of facial stimulation and the expertise of the examinees. These factors are concluded from some relevant researches. However, it is unknown what the relationships of these factors are and how they work.

Eastwood, Smilek and Merikele (2001) used sight search operation study to detect positive and negative emotional faces in quiet faces and found that when the faces are upright, the detection rate of positive emotional faces are higher than that of negative emotional faces while when the faces of upside-down, there is no difference.

It could be found from the above-mentioned researches that the direction of faces exerts great influences over face recognition. When upright, it could quickly obtain holistic information while when upside-down, the information could not be acquired. 


\section{Purposes}

The previous researches indicate that smile face is the expression that is not easy to be mixed up with other expressions (Kirita T and Endo WI1993). When upside-down, the recognition of smiling faces is more greatly influenced. What is more, compared with other expression, in the recognition process of smiling face, the examinees rely more on the facial holistic recognition strategy (Kirita T and Endo WI (1993).). This paper would further discuss this conclusion. Psychologists and humanists all admit that facial expressions have universality and they are, to some extent, cross-cultural and cross-national. Wherever, smiling faces are to express happiness. We need to explore whether human beings more adopt holistic recognition strategy even within cross-cultural context by taking into consideration of the universality of smiling face cognition and applying the stimulation conditions of upside-down faces to present pictures

Based on the researches on Chinese and Japanese examinees, this paper did researches on the smiling face memory cognition on foreigners' pictures and native people's pictures. The research subjects include, firstly, whether smiling face memory cognition are more greatly influenced by upside-down effect; whether smiling face cognition more rely on holistic configurable information; under the condition of upside-down, whether partial feature recognition strategy is more advantaged; secondly, whether holistic recognition strategy could overcome difficulties of recognizing various particular facial expressions of people from different cultures and whether the smiling face could be commonly shared among the different cultures; thirdly, under the condition of upright, compared with quiet face, whether the response time of smiling face is shorter and the recognition degree of smiling face is higher.

Hence, the following assumptions are made: firstly, due to the advantages of smiling face cognition, when the examination series pictures are upright, for native examinees, no matter it is native people's pictures or foreigners' pictures, the response time of smiling face should be shorter than that of quiet face and the recognition degree of smiling face should be higher than that of quiet face. On the contrary, when the examination series pictures of upside-down, the response time of smiling face should be longer than that of quiet face and the recognition degree of smiling face should be lower than that of quiet face; secondly, the upside-down effect should be proved.

\section{Objects and methodology}

\subsection{Examinees}

The examinees are twenty-four undergraduates and postgraduates (12 Chinese and 12 Japanese; 12 males and 12 females).

\subsection{Methodology}

\subsubsection{Preparation experiment}

The preparation experiment adopted expression pictures of real face stimulation.

\subsubsection{Evaluators}

The evaluators are ten undergraduates and postgraduates ( 5 males and 5 females).

\subsubsection{Preparation experiment procedures}

We took pictures for 198 Chinese and Japanese undergraduates (97 males and 101 females) with 2 expressions for each student (smiling face and quiet face). The Evaluators evaluated every face pictures. They used six items (happy, angry, sad, surprised, confused and quiet and adopted Likert self-evaluation seven points measurement table to make score (1. completely did not express at all; 2. comparatively did not express; 3 . did not express to some extent; 4. not sure; 5; expressed somewhat; 6: comparatively expressed; 7: fully expressed) so as to evaluate each facial picture. They evaluated the expression degree of each picture. For each expression, we use the average value of the dimension values of each evaluator as the final evaluation result. Then, we compared the average values of each expression picture and the higher ones are considered as the expression of pictures.

We adopted pictures of 32 students who well expressed their smiling face and quiet face as the target pictures of this experiment. In the left students' pictures, we selected from the two expressions the one that fully expresses their expressions as the interruption pictures. (32 students).

\subsubsection{Experiment location}

The experiment location is Advanced Psychology Laboratory of the Education Department of Kagoshima University.

\subsubsection{Experiment materials}

The smiling face and quiet face pictures of 64 people ( 32 males and 32 females). The examination series pictures are of eight types of combination, (smiling face, quiet face $) \times($ Chinese, Japanese $) \times($ upright, upside-down). The total number is 32. They were inputted into the computer and are made into stimulation pictures. When conducting recognition memory test experiment, the conditions for expression changes are that half of the target expressions are different from the examination series pictures and the other half are the same with examination series pictures. What is more, in the 
recognition test, all pictures are upright. We selected 8 pictures for each expression from the Chinese and Japanese interrupting pictures (32 pictures). The total number of target pictures and interrupting pictures are 64 . They are input into computer and are made into pictures for recognition memory test experiment.

\subsubsection{Experiment procedures}

Examination series pictures include the (target people's) expressions, the stimulation pictures' direction and nationality 32 stimulation pictures on computer screen. The distance between the examinee and the screen is $28.7 \mathrm{~cm}$. The size of the pictures is sight angle vertical direction $23.5^{\circ} \times$ horizontal $21^{\circ}$.

Each examinee is instructed as follows:

Welcome to attend face recognition experiment. We will show you some male and female's smiling face and quiet face pictures, either upright or upside-down. The show time of each picture is 4 seconds. Please carefully view each picture and memorize them. After the first facial picture stimulation recognition experiment, we will give each of you a piece of paper and please randomly draw a tree within 5 minutes. After that, we will begin to conduct the recognition memory test experiment. The screen will again show facial pictures and among those pictures, some are people that you remember and some are people you have not seen in the first experiment. What is more, the all pictures in the second recognition memory test experiment are al upright pictures. Please notice that the pictures have nothing to do with their expression and please just decide whether they are the same person. When the picture shown in the second time is the same person as the first time, please press $\mathrm{E}$ as soon as possible. If not, please press I as soon as possible. After pressed, the recognition memory test experiment is over. Your response is better if quicker. When the experiment starts, please put your right hand and left hand middle figure right on the button $\mathrm{E}$ and $\mathrm{I}$.

We conducted the experiment according to the following orders (table 1). When doing recognition memory test experiment, after the screen shows pictures, after the examinees pressed the button the picture disappearance and the response button and time are recorded. If no response within 3 seconds, the picture will disappear and this test is considered as non-effective response (Rhodes, 1989).

\section{Results}

We calculated 24 examinees' average recognition response time and average recognition correctness's d' values in the recognition memory test experiment (the calculation of the $\mathrm{d}^{\prime}$ value of recognition correctness applies signal measurement theory), compared the examination series pictures expression (Quiet, Smiling face) $\times$ Nationality (Chinese picture and Japanese picture) $\times$ direction (upright, upside-down) and used $2 \times 2 \times 2$ to do variance analysis.

\subsection{The average recognition response time of recognition memory test experiment}

4.1.1 In the recognition response time, in Japanese examinees group, the main effect of the cross-function between expression and directions of the examination series pictures are remarkable $(F(1,35)=3.616, p<0.05)$.

After doing simple main effect analysis, it is found that the examination series pictures' expressions and upright directions have remarkable difference, $p<0.05$. What is more, the direction and quiet facial expression have remarkable difference, $p<0.05$. Please refer to picture 1 and picture 2 for the experimental results.

For recognition response, in Japanese examinees group, no matter it is Japanese pictures or Chinese pictures, the analysis results for cross-function between the examination series pictures' expression and direction show that when the stimulation pictures are upright, compared with quiet face, the response time of smiling face is shorter. On the contrary, when the stimulation pictures are upside-down, compared with quiet face, the response time of smiling face is longer.

4.1.2 In Chinese examinees group, the main effect of the cross-function between examination series pictures' expression and direction is remarkable $(F(1,35)=4.383, p<0.05)$. The simple main effect analysis results are the same as the Japanese examinees group. What is more, in recognition response time, in the Chinese examinees group, no matter they are Japanese pictures or Chinese pictures, the analysis results of the cross-function between the examination series pictures' expression and directions are the same as that of the Japanese examinees group. Please refer to picture 3 and picture 4 for the experiment results.

\subsection{The $d^{\prime}$ value of the average recognition correctness in recognition memory test experiment}

4.2.1 In the $\mathrm{d}^{\prime}$ value of the recognition correctness, in the Japanese examinees group, the main effect of the cross-function between examination series pictures' expression and direction is remarkable $(F(1,3)=11.626, p<0.05)$. After doing simple main effect analysis, it is found that the examination series pictures' expressions and upright directions have remarkable difference, $p<0.05$. What is more, the direction and quiet facial expression have remarkable difference, $p<0.05$; the direction and smiling face have remarkable difference, $p<0.05$. In addition, the main effect of the direction is remarkable $(F(1,3)=58.80, p<0.005)$. Please refer to picture 5 and picture 6 for the experimental results.

For $\mathrm{d}^{\prime}$ value of the recognition correctness, in Japanese examinees group, no matter they are Japanese pictures or Chinese pictures, the analysis results for cross-function between the examination series pictures' expression and 
direction show that when the stimulation pictures are upright, compared with quiet face, the recognition correctness of smiling face is higher. On the contrary, when the stimulation pictures are upside-down, compared with quiet face, the recognition correctness of smiling face is lower. All in all, when the stimulation pictures are upright, it promotes the recognition correctness of smiling face picture recognition to rise. Therefore, the advantages of smiling face are determined. When the stimulation pictures are upside-down, the recognition correctness of quiet faces rises and turned into the result of the advantages of quest face.

4.2.2 In Chinese examinees group, the main effect of the cross-function between examination series pictures' expression and direction is remarkable $(F(1,35)=12.945, p<0.05)$. The simple main effect analysis results are the same as that of the Japanese examinees group. What is more, in $\mathrm{d}^{\prime}$ value of the recognition correctness, in the Chinese examinees group, no matter they are Japanese pictures or Chinese pictures, the analysis results of the cross-function between the examination series pictures' expression and directions are the same as that of the Japanese examinees group. Please refer to picture 7 and picture 8 for the experiment results.

\section{Discussion}

It could be seen from the experimental results that in the recognition response time, the Japanese examinees group and Chinese examinees group got the same conclusion; in the $\mathrm{d}^{\prime}$ value of the recognition correctness, he Japanese examinees group and Chinese examinees group also got the same conclusion.

When the simulation pictures are upright, the recognition of smiling face pictures promotes the recognition response time. Therefore, the advantage of the smiling face is determined. When the stimulating pictures are upside-down, the recognition response time of quiet face becomes shorter and it came the result of the advantage of quiet face. Therefore, the upside-down effect is proved. What is more, from the above resulted, it could be drawn that the recognition response time for native pictures is shorter than that of foreigners' pictures. To put it in other way, the native facial pictures are more remarkable than that of foreigners' facial pictures.

When the simulation pictures are upright, the recognition of smiling face pictures promotes the recognition correctness. Therefore, the advantage of the smiling face is determined. When the stimulating pictures are upside-down, the recognition correctness of quiet face rises and it came the result of the advantage of quiet face. Therefore, the upside-down effect is proved. What is more, from the above resulted, it could be drawn that the recognition correctness for native pictures is higher than that of foreigners' pictures. To put it in other way, the native facial pictures are more remarkable than that of foreigners' facial pictures.

The two assumptions of this research are proved.

Through the experimental results, we cold conclude that:

Firstly, for smiling face effect, when the examination series pictures are upright, compared with quiet face, the response time of smiling face is shorter and the recognition correctness of smiling is higher.

Secondly, it is hard for the holistic recognition strategy to exert its influence when upside-down.

Thirdly, for upside-down effect, the experimental results show that phenomenon the recognition of upside-down faces is harder than that of upright face complies with the face upside-down effect raised by Yin (1969).

The fourth is the difference between native and foreigners' face. For native face memory cognition, the smiling face effect is obvious (Chinese examinees show Chinese face pictures; Japanese examinees use Japanese face pictures). For foreigners' face memory cognition, the smiling face effect is week (Chinese examinees use Japanese face pictures; Japanese examinees use Chinese face pictures).

All in all, the cognition of smiling face is more influenced by upside-down effect. Hence, it shows that the recognition of smiling face more relies on holistic recognition strategy. It could further be believed that for upright faces, the holistic recognition strategy has advantages while for upside-down faces, partial feature recognition strategy has advantages. What is more, holistic recognition strategy could overcome difficulties of recognizing various particular facial expressions of people from different cultures, and the smiling face could be commonly shared between the different cultures. 


\section{References}

Carey S, Diamond R. (1977). From piecemeal to configurational representation of faces. Science, 195:312-314.

Eastwood JD, Smilke D \& Merikle PM. (2001). Differential attentional guidance by unattended faces expressing positive and negative emotion. Percept psychophys, 63:1004-1013.

Kirita T \& Endo WI. (1993). Facial cognition process: discussion on the effect of expression. Japanese psychology institute, 57: 522-526.

Kirita T \& Endo WI. (1992). Face recognition process: expression judgment. Japanese psychology institute, 56: $247-250$.

Maja, Pantic, Leon J.M.Rothkrantz. (2002). Automatic analysis of facial expressions: the state of the art, IEEE Trans. On pattern analysis and machine intelligence, 2 (12).

Rhodes G, Brake S \& Taylor K,et al. (1989). Expertise and configural coding in face recognition. Bri j psychol, 80:313-331.

Valentine T, \& Bruce V. (1988). Mental rotation of faces. Mem cogn, 16: 556-566.

Yin, RK. (1969). Looking at upside-down faces. J exp psychol, 81: 141-145.

Yin, RK. (1970). Face recognition by brain-injured patientsa dissociable ability. Neuropsyecholgia, 8:395-402. 
Table 1. Simulation appearances order table

\begin{tabular}{|c|c|c|c|}
\hline $\begin{array}{c}\text { Examination series } \\
\text { pictures }\end{array}$ & $\begin{array}{l}\text { Recognition memory test } \\
\text { pictures }\end{array}$ & Pieces & Interrupting pictures \\
\hline \multirow[t]{2}{*}{ Japanese Picture } & $\begin{array}{l}\text { Japanese Picture } \\
\text { (Upright, Quiet) }\end{array}$ & 2 & \\
\hline & $\begin{array}{c}\text { Japanese Picture } \\
\text { (Upright, Smiling face) }\end{array}$ & 2 & \\
\hline \multirow[t]{2}{*}{$\begin{array}{c}\text { Japanese Picture } \\
\text { (Upside-down, Quiet) }\end{array}$} & $\begin{array}{l}\text { Japanese Picture } \\
\text { (Upright, Quiet) }\end{array}$ & 2 & \\
\hline & $\begin{array}{c}\text { Japanese, Picture } \\
\text { (Upright, Smiling face) }\end{array}$ & 2 & \\
\hline \multirow[t]{2}{*}{$\begin{array}{l}\text { Chinese Picture } \\
\text { (Upright, Quiet) }\end{array}$} & $\begin{array}{l}\text { Chinese Picture } \\
\text { (Upright, Quiet) }\end{array}$ & 2 & $\begin{array}{c}\text { Japanese Picture } \\
\text { (Upright, Quiet)Eight pictures }\end{array}$ \\
\hline & $\begin{array}{c}\text { Chinese Picture } \\
\text { (Upright, Smiling face) }\end{array}$ & 2 & \\
\hline \multirow[t]{2}{*}{$\begin{array}{c}\text { Chinese Picture } \\
\text { (Upside-down, 'Quiet) }\end{array}$} & $\begin{array}{l}\text { Chinese Picture } \\
\text { (Upright, Quiet) }\end{array}$ & 2 & $\begin{array}{c}\text { Japanese Picture } \\
\text { (Upright, Smiling face) } \\
\text { Eight pictures }\end{array}$ \\
\hline & $\begin{array}{c}\text { Chinese Picture } \\
\text { (Upright, } \cdot \text { Smiling face) }\end{array}$ & 2 & \\
\hline \multirow[t]{2}{*}{$\begin{array}{c}\text { Japanese Picture } \\
\text { (Upright, } \cdot \text { Smiling face) }\end{array}$} & $\begin{array}{c}\text { Japanese Picture } \\
\text { (Upright } \cdot \text {, Smiling face) }\end{array}$ & 2 & $\begin{array}{l}\text { Chinese Picture } \\
\text { (Upright, Quiet) Eight pictures }\end{array}$ \\
\hline & $\begin{array}{l}\text { Japanese Picture } \\
\text { (Upright, Quiet) }\end{array}$ & 2 & \\
\hline \multirow[t]{2}{*}{$\begin{array}{l}\text { Japanese Picture } \\
\text { (Upside-down, } \\
\text { Smiling face) }\end{array}$} & $\begin{array}{c}\text { Japanese Picture } \\
\text { (Upright, } \cdot \text { Smiling face) }\end{array}$ & 2 & $\begin{array}{c}\text { Chinese Picture } \\
\text { (Upright·, Smiling face) } \\
\text { Eight pictures }\end{array}$ \\
\hline & $\begin{array}{l}\text { Japanese Picture } \\
\text { (Upright·, Quiet) }\end{array}$ & 2 & \\
\hline \multirow[t]{2}{*}{$\begin{array}{c}\text { Chinese Picture } \\
\text { (Upright, } \cdot \text { Smiling face) }\end{array}$} & $\begin{array}{c}\text { Chinese Picture } \\
\text { (Upright, } \cdot \text { Smiling face) }\end{array}$ & 2 & \\
\hline & $\begin{array}{l}\text { Chinese Picture } \\
\text { (Upright, ·Quiet) }\end{array}$ & 2 & \\
\hline \multirow[t]{2}{*}{$\begin{array}{c}\text { Chinese Picture } \\
\text { (Upside-down, Smiling } \\
\text { face) }\end{array}$} & $\begin{array}{c}\text { Chinese Picture } \\
\text { (Upright, } \cdot \text { Smiling face) }\end{array}$ & 2 & \\
\hline & $\begin{array}{l}\text { Chinese Picture } \\
\text { Upright·, Quiet) }\end{array}$ & 2 & \\
\hline
\end{tabular}




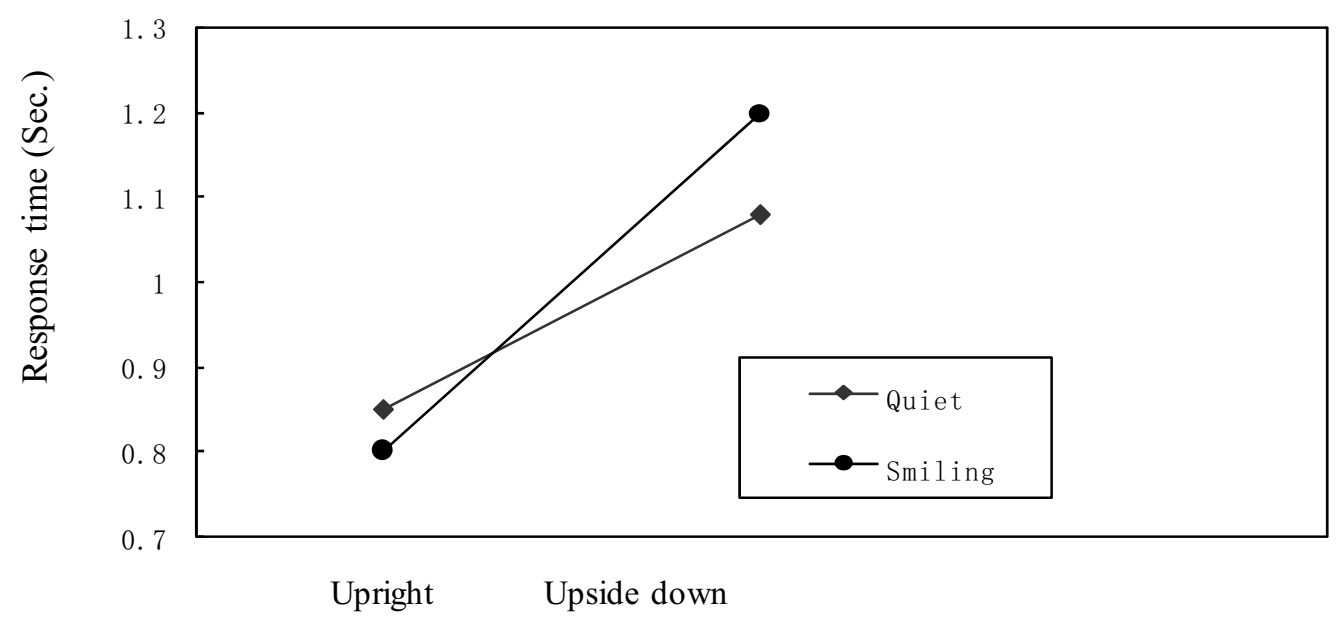

Figure 1. Comparison of the response time of Japanese Pictures (Examinees: Japanese)

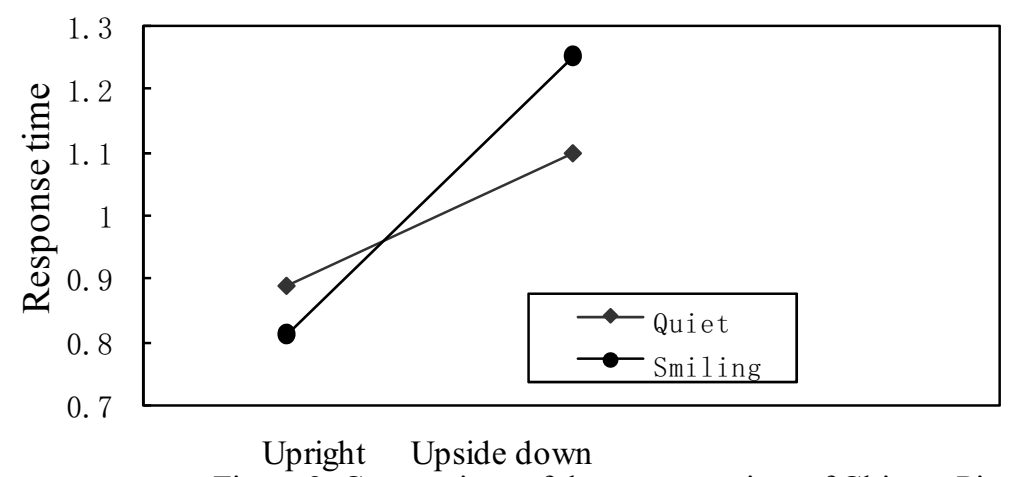

Figure 2. Comparison of the response time of Chinese Pictures (Examinees: Japanese) 


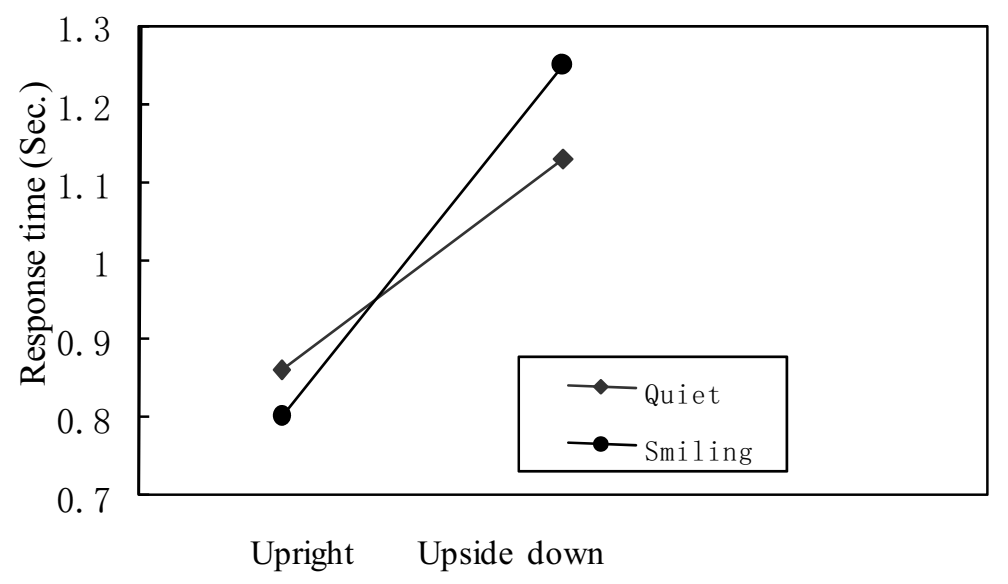

Figure 3. Comparison of the response time of Chinese Pictures (Examinees: Chinese)

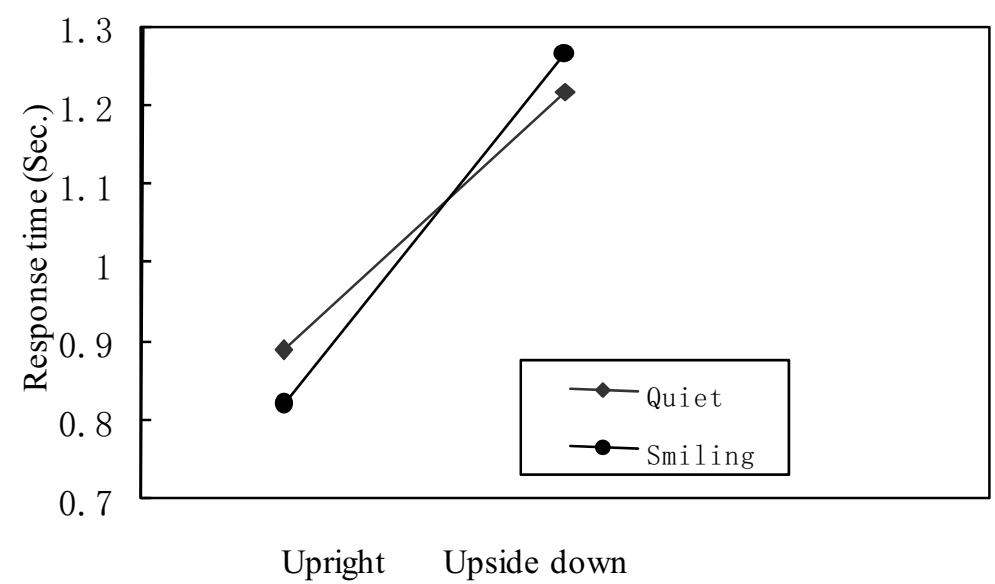

Figure 4. Comparison of apanese Pictures (Examinees: Chinese)

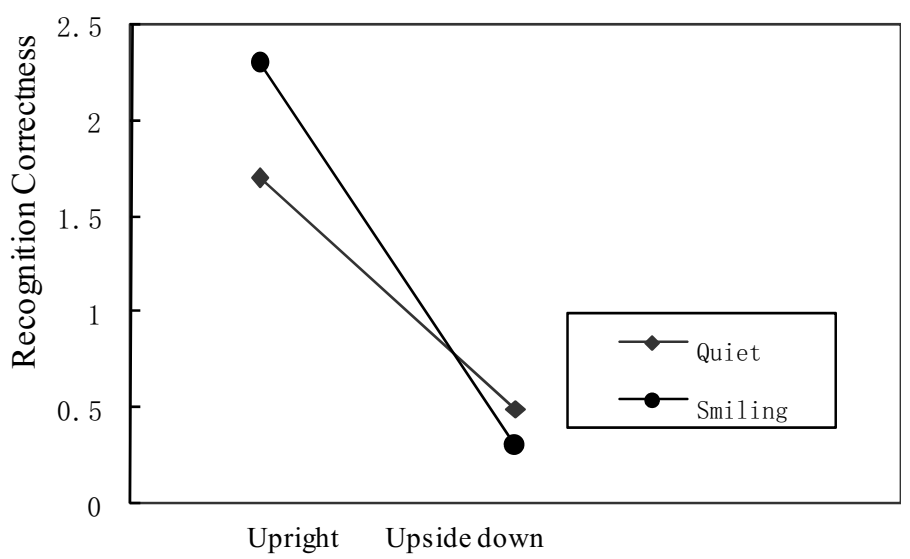

Figure 5. Comparison of the d' value of Japanese Pictures (Examinees: Japanese) 


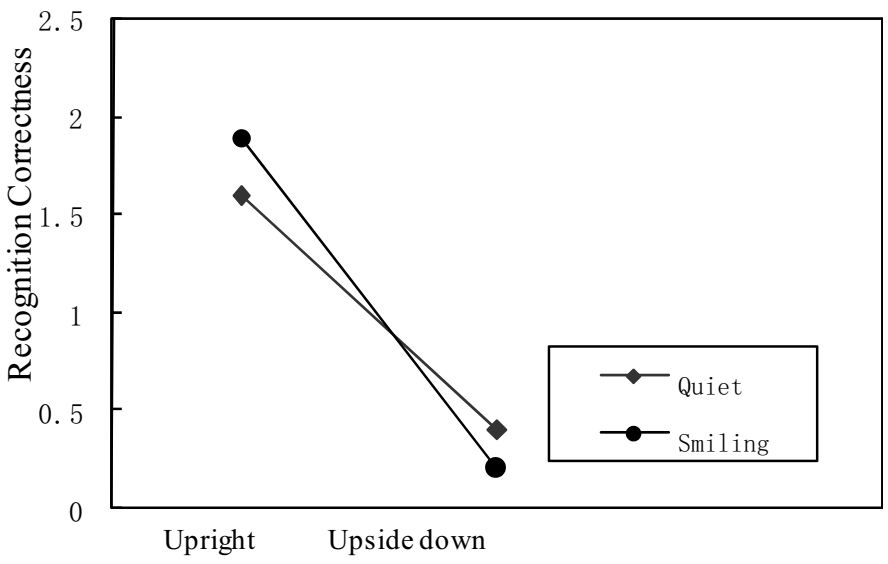

Figure 6. Comparison of the $d^{\prime}$ value of Chinese Pictures (Examinees: Japanese)

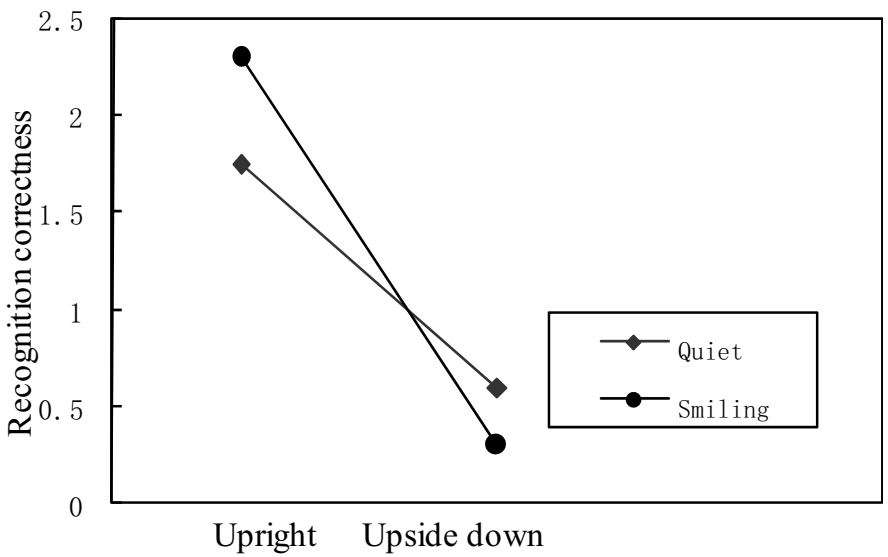

Figure 7. Comparison of the $\mathrm{d}^{\prime}$ value of Chinese Pictures (Examinees: Chinese)

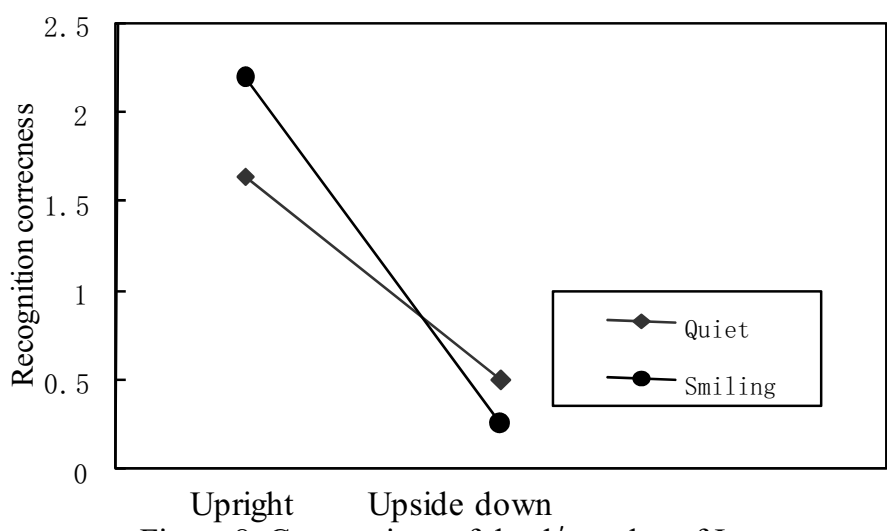

Figure 8. Comparison of the $\mathrm{d}^{\prime}$ value of Japanese Pictures (Examinees: Chinese) 